\title{
Large array of low-frequency readout quantum capacitance detectors
}

\author{
Pierre M. Echternach,* Andrew D. Beyer, and Charles M. Bradford \\ Jet Propulsion Laboratory, California Institute of Technology, Pasadena, California, \\ United States
}

\begin{abstract}
Quantum capacitance detectors (QCDs) are photon shot noise-limited terahertz detectors based on a single Cooper-pair box superconducting qubit. The QCD has demonstrated photon shot noise-limited performance for $1.5 \mathrm{THz}$ radiation under loading conditions between $10^{-20}$ and $10^{-18} \mathrm{~W}$ and single-photon detection and counting at that frequency. We report here fabrication and preliminary characterization of a 441 pixel array of QCDs with readout frequencies between 700 and $850 \mathrm{MHz}$. (C) The Authors. Published by SPIE under a Creative Commons Attribution 4.0 Unported License. Distribution or reproduction of this work in whole or in part requires full attribution of the original publication, including its DOI. [DOI: 10.1117/1.JATIS.7.1.011003]
\end{abstract}

Keywords: quantum capacitance; detector; far-infrared; terahertz.

Paper 20077SS received Jun. 15, 2020; accepted for publication Dec. 9, 2020; published online Jan. 6, 2021.

\section{Introduction}

The Origins Space Telescope (Origins) traces our cosmic history, from the formation of the first galaxies and the rise of metals to the development of habitable worlds and present-day life. Origins does this through exquisite sensitivity to infrared radiation from ions, atoms, molecules, dust, water vapor, and ice, and observations of extra-solar planetary atmospheres, protoplanetary disks, and large-area extragalactic fields. Origins operates in the wavelength range 2.8 to $588 \mu \mathrm{m}$ and is more than 1000 times more sensitive than its predecessors due to its large, cold (4.5 K) telescope and advanced instruments. ${ }^{1}$

There are a number of approaches to far-IR and sub-mm photodetection using superconducting devices that are being explored as possible technologies to obtain the very low-noise equivalent powers (NEPs of about $10^{-20} \mathrm{~W} / \mathrm{Hz}^{1 / 2}$ ) required for optimal use of the low-background platform. One such technology, the kinetic inductance detector, ${ }^{2}$ relies on the sensitivity of the surface inductance to the presence of pair-breaking radiation. Two others, the transition edge sensor $^{3-5}$ and the nano-hot electron bolometer (nano-HEB), ${ }^{6}$ rely on low critical temperature and high thermal isolation to achieve high sensitivity.

The approach at hand, the quantum capacitance detector $(\mathrm{QCD}),{ }^{7-10}$ is based on the extreme sensitivity of the single Cooper-pair box (SCB), a mesoscopic superconducting device, to pair breaking radiation. In a QCD, a single quasiparticle tunneling event produces a frequency shift comparable to or larger than a resonator linewidth. QCDs have demonstrated photon shot noiselimited performance with useful optical efficiency ${ }^{11}$ for optical loadings between $10^{-20}$ and $10^{-18} \mathrm{~W}$, corresponding to an NEP below $10^{-20} \mathrm{~W} / \mathrm{Hz}^{1 / 2}$. This sets the QCD apart from the other approaches, which have to date not demonstrated NEPs lower than $0.95 \times 10^{-19} \mathrm{~W} / \mathrm{Hz}^{1 / 2}$ in a single pixel demonstration or $2 \times 10^{-19} \mathrm{~W} / \mathrm{Hz}^{1 / 2}$ in a kilopixel demonstration. ${ }^{12-14}$ Another important attribute of the QCDs is its speed-the devices are intrinsically fast, and the combination of sensitivity and speed enables photon counting. In an experiment using a fast readout, the QCD has demonstrated $1.5 \mathrm{THz}(200 \mu \mathrm{m})$ photon counting under a range of loadings. ${ }^{15}$ Although not required for much of Origins science, a true thresholding photon-counting device is not susceptible to some of the systematic noise concerns of a total-power detector, in particular low-audio-frequency noise due to amplifiers, electronics, or resonators themselves.

*Address all correspondence to Pierre M. Echternach, pierre.m.echternach@jpl.nasa.gov 


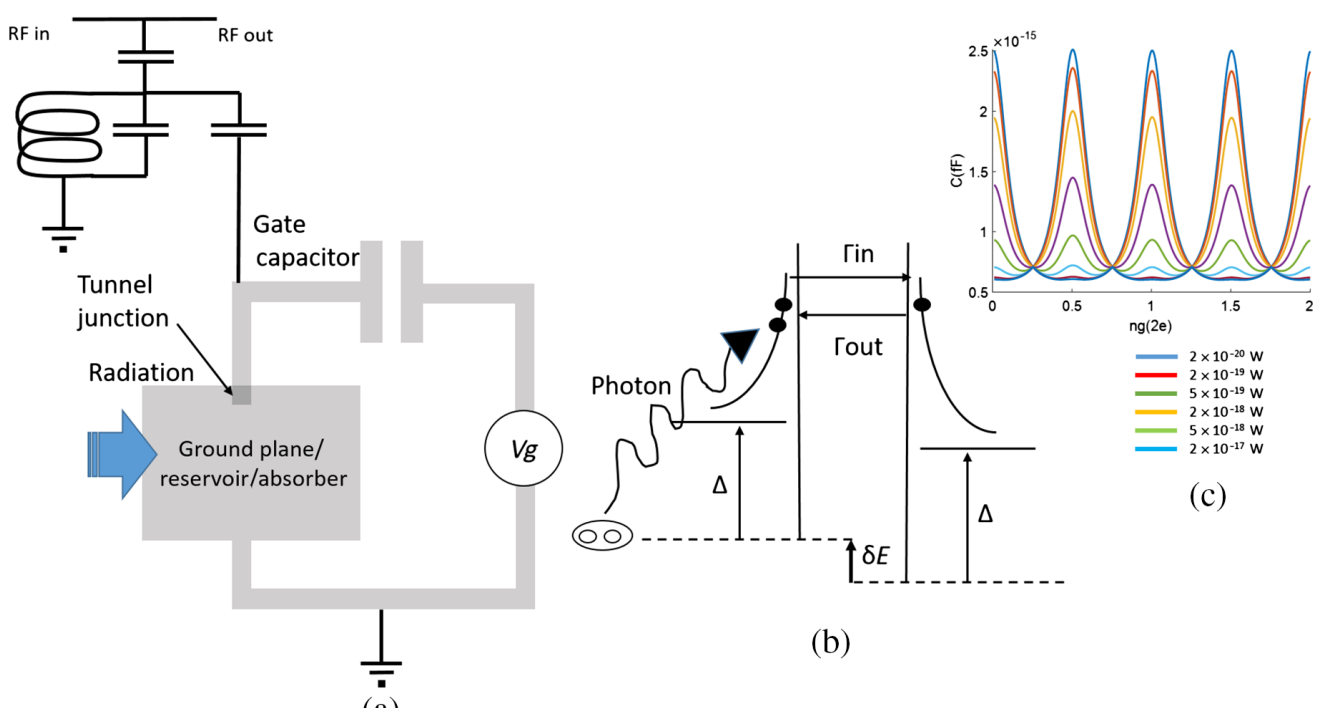

Fig. 1 (a) Schematic diagram of a QCD. (b) Energy diagram of single Cooper-pair box at the degeneray point $\left(C_{g} V_{g} / e=1\right)$ showing photogeneration of a quasiparticle and tunneling rates in and out of the island. (c) Simulation of quantum capacitance signal for various illumination levels.

\section{Principle of Operation}

In the general QCD design, shown in Fig. 1(a), radiation is absorbed in an antenna coupled or mesh absorber connected to an island of superconducting material via a small $(100 \times 100 \mathrm{~nm})$ tunnel junction defining a device dubbed the SCB. The SCB is embedded in a resonator. Quasiparticles trapped in the reservoir can only tunnel in and out of the island, changing its capacitance. This capacitance change causes a shift in the resonance frequency of the resonator and can be measured by the phase shift of an RF signal transmitted through a feedline that is capacitively coupled to the resonator. This design allows for straightforward frequency multiplexing with each pixel having a unique resonant frequency, and all resonators coupled to a single feedline.

The operation of the QCD is sketched in Fig. 1(b). Radiation coupling to the absorber via the antenna breaks Cooper pairs in the absorber, generating quasiparticles that can tunnel in and out of a small island that is capacitively coupled to the readout resonator. The rate of tunneling from the absorber into the island is proportional to the quasiparticle population $N_{q p}$ in the absorber so $\Gamma_{\text {in }}=K N_{q p}$, where $K$ is a constant which depends on the tunnel junction parameters and temperature. ${ }^{7}$ The rate of tunneling out of the island back to the absorber, on the other hand, is largely independent of the quasiparticle population in the absorber. The probability of occupation of the island by a single quasiparticle is $P_{\text {odd }}=\Gamma_{\text {in }} /\left(\Gamma_{\text {in }}+\Gamma_{\text {out }}\right)$. When the gate voltage is swept, one observes a series of peaks in the capacitance of the island ${ }^{16}$ with an average amplitude given by $\Delta C=\left(1-P_{\text {odd }}\right)\left(4 E_{C} / E_{J}\right) C_{g}^{2} / C_{\Sigma}$, where $E_{c}$ is the junction charging energy $e^{2} / 2 C_{\Sigma}$, $E_{J}$ is the junction's Josephson energy, $C_{\mathrm{g}}$ is the gate capacitor capacitance, and $C_{\Sigma}$ is the sum of the gate and junction capacitances. Since $\Delta C$ is a function of $N_{q p}$, and $N_{q p}$ is a function of the optical signal power coupled by the antenna, a measurement of $\Delta C$ is a measurement of the optical signal power [Fig. 1(c)]. The change in capacitance is measured by the phase shift of the RF signal transmitted through the feedline, which is caused by the change in the resonant frequency of the resonator.

\section{Device Description}

We fabricated a $21 \times 21$ array of pixels in a square grid with $0.9 \mathrm{~mm}$ spacing [Fig. 2(b)]. A feedline meanders through the array to couple the readout signal to each resonator. The devices 


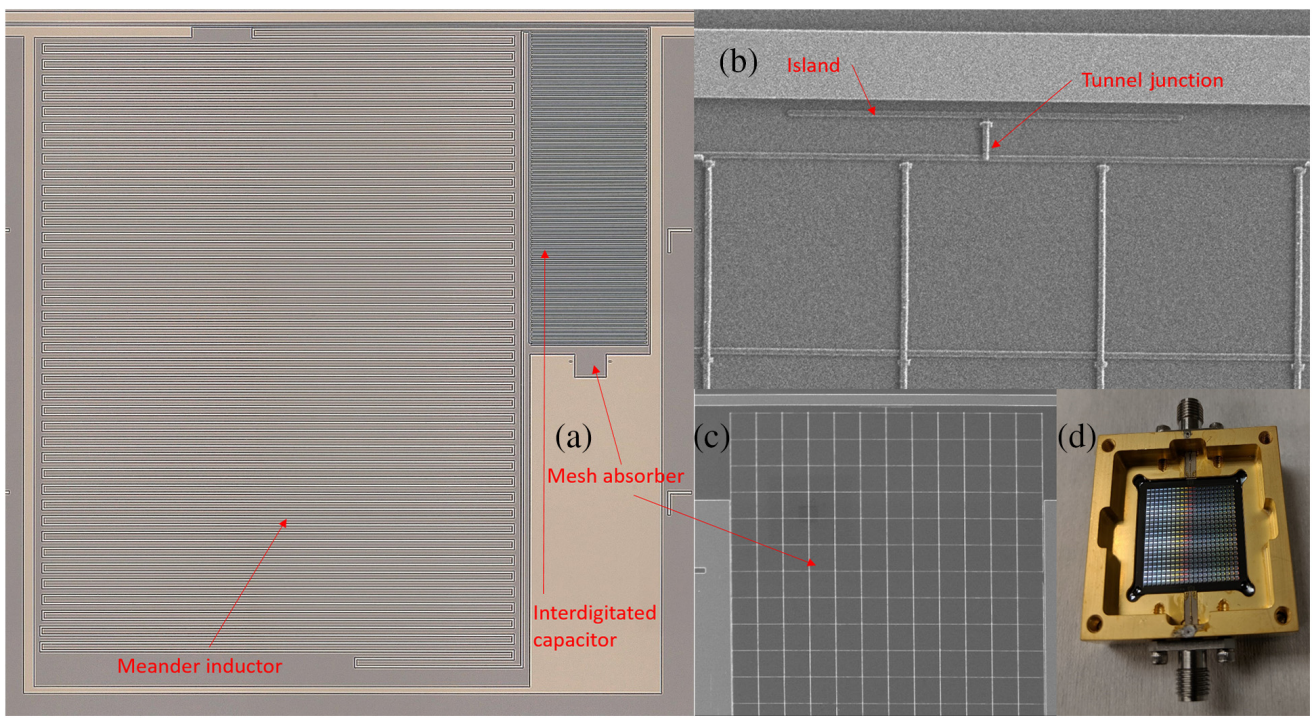

Fig. 2 (a) $21 \times 21$ array of QCDs. (b) SEM picture of single Cooper-pair box showing the island in close proximity to the lowest finger of the interdigitated capacitor, the tunnel junction, and a portion of the mesh absorber.

are formed from thin-film superconducting metals patterned on a high-resistivity float-zone silicon substrate $500-\mu \mathrm{m}$-thick. The resonators are niobium films patterned into meandered inductors and interdigitated capacitors [Fig. 2(a)], with the frequency set for different devices by variations in the inductor length. Each absorber is a $60 \mu \mathrm{m} \times 60 \mu \mathrm{m}$ aluminum mesh consisting of $100 \mathrm{~nm}$ wide, 20-nm-thick aluminum lines on a grid with unit cell $5 \mu \mathrm{m} \times 5 \mu \mathrm{m}$ as shown in Fig. 2(c); the resulting total volume of aluminum is $1.56 \mu \mathrm{m}^{3}$. The absorbers are backsideilluminated. In the previous devices $(5 \times 5$ arrays $)$, radiation was focused onto the meshes with an array of Fresnel lenses, also etched in a high-resistivity float-zone silicon substrate. Since at the time of the measurements described here we did not have a matching $21 \times 21$ array of Fresnel lenses, we have simply used a flat piece of silicon as a backing plane.

In order to maximize absorption, the absorber's sheet resistance should be set to $377 /(n-1) \Omega / \square,{ }^{17}$ where $n=3.4$ is the index of refraction of the substrate. The sheet resistance of diagnostic mesh absorbers placed on the same chip measured at $4.2 \mathrm{~K}$ was $72 \Omega / \square$ somewhat lower than the target. Electromagnetic simulations indicate that this should yield an absorption efficiency of $49 \%$ at $1.5 \mathrm{THz}$.

The SCB island is a T-shaped line in close proximity to the lowermost finger of the resonator interdigitated capacitor [Fig. 2(b)]. The tunnel junction is formed at the overlap between the vertical part of the island and the mesh absorber.

\section{Measurements}

\subsection{Measurement Approach}

To characterize their performance, the samples were cooled in a dilution refrigerator, mounted inside a shield at the mixing chamber temperature. The optical set up is shown in Fig. 3(a). A cylindrical opening (baffle) on the mixing chamber contains a stack of three mesh filters with a $10 \%$ bandwidth around $1.5 \mathrm{THz}$ and one zytex filter to block radiation at wavelengths smaller than the mesh hole size. The cylinder housing the filter stack is coated inside and out with a black material composed of a mixture black epoxy and lamp black carbon ${ }^{18}$ and acts to limit the field of view of the detector array to a small window at the still temperature. This material, referred to as "Berkeley Black," has a reported reflectivity ranging from 0.1 for a wavelength of $1 \mathrm{~mm}$ to 0.025 at $167 \mu \mathrm{m}$ wavelength. ${ }^{18}$ The mixing chamber shield with its inside surface coated by the same black material is inside the still shield. The still shield has baffles around the mixing chamber shield cylindrical opening. The inside of the still shield was coated with the black material and so 


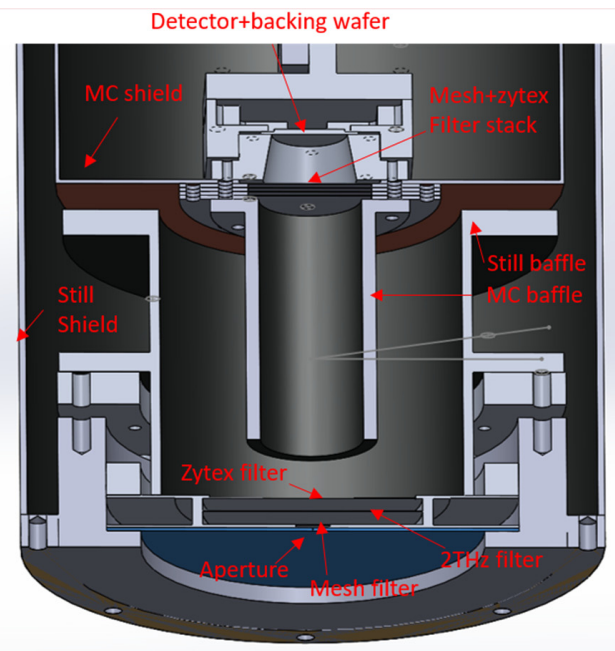

(a)

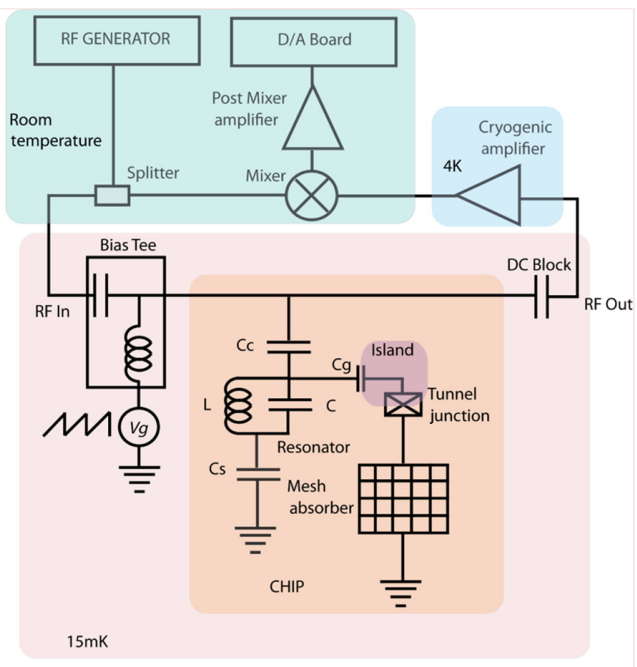

(b)

Fig. 3 (a) Schematic diagram of QCD readout. (b) Schematic diagram of optical characterization setup.

were the baffles (inside and out). The array was illuminated through a $500-\mu \mathrm{m}$ aperture by a black body source outside of the still shield and thermally regulated with a weak thermal link to the helium bath. An additional mesh filter is placed behind the aperture followed by a 2-THz low-pass filter and an additional zytex filter. The transmission coefficients of the optical filters were measured using a Fourier-transform infra-red spectrometer and some results are shown in Fig. 4. The black curve is the measurement for a single mesh filter. The blue curve is the measured transmission for a stack of three mesh filters and it shows that the curve shoulders are at the noise floor of the spectrometer. For comparison, we plot in red the product of the transmissions of three filters showing the net transmission falls below the noise floor of the instrument. The actual filter stack contains a total of four mesh filters, one $2 \mathrm{THz}$ low-pass filter and two zytex filters. The product of the transmissions is shown in cyan. A model function (shown in green) was used in the actual power calculations.

Radiation is usually focused on the individual pixels by an array of Fresnel lenses fabricated on high-resistivity float-zone silicon, the same substrate used for the detectors. Since for the

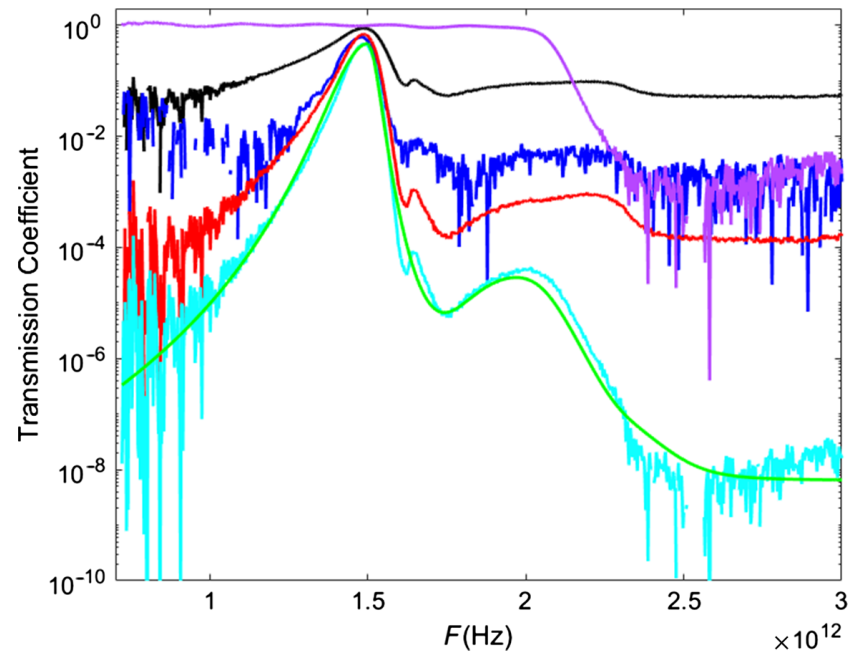

Fig. 4 Transmission coefficients of the filter stack. Black: a single mesh filter (measurement). Blue a stack of three mesh filters (measurement). Red: the product of three mesh filters (from the blue curve). Purple: 2-THz low-pass filter (measurement). Cyan: product of the transmission coefficients of all filters in the filter stack. Green: model function used to calculate the optical power. 
devices described here we did not have a matching lens array, we have used a flat piece of silicon as a holder. The devices are still back side illuminated. The radiation absorbed is calculated as

$$
P s=\epsilon_{\mathrm{mesh}} \Omega_{M} A_{A} \int_{v_{l}}^{\nu_{h}} \frac{T(\nu) h \nu^{3} / c^{3}}{e^{\frac{h \nu}{k_{B} T}}-1} \mathrm{~d} \nu,
$$

where $T(\nu)$ is the transmission through the filter stack, $\varepsilon_{\text {mesh }}$ is the calculated mesh absorption efficiency, $\Omega_{M}$ is the solid-angle subtended by mesh absorber as seen from the aperture, and $A_{A}$ is the aperture area. The lower limit of integration was taken as $100 \mathrm{GHz}$, the lowest frequency that can break a Cooper pair in 20-nm-thick aluminum films, which have a critical temperature of $\sim 1.36 \mathrm{~K}$, as measured in the previous samples with the same thickness. The highest limit of integration was taken as $2 \mathrm{THz}$, since the $2 \mathrm{THz}$ filter provided a very sharp cutoff at that frequency.

As shown in Fig. 3(b), measurements are performed by sending an RF signal at the resonant frequency of the QCD resonator through a feedline which is capacitively coupled to the resonator. The transmitted signal is amplified by a cryogenic amplifier and is down-converted by a mixer, yielding a voltage proportional to the phase shift acquired by the RF signal. The resulting signal is digitized and recorded. To characterize the performance we sweep the gate voltage with a sawtooth waveform at a frequency of $1000 \mathrm{~Hz}$ and amplitude encompassing 5 quantum capacitance peaks.

\subsection{Results}

Figure 5(a) shows the transmission through the device. Of the 441 design frequencies, 416 were found. The absent frequencies may have a dip too small to observe or there may be collisions with other frequencies.

To estimate the NEP, for each optical loading, we have measured the quantum capacitance time stream for $0.5 \mathrm{~s}$ while sweeping the gate voltage at $1000 \mathrm{~Hz}$ with an amplitude of $1.5 \mathrm{~V}$, enough to sweep over 5 quantum capacitance peaks. We calculated the standard deviation of the time stream, which will be proportional to the amplitude of the quantum capacitance signal (plus additional noise). We repeated the measurement 125 times, and estimated the standard deviation of the results, which is our RMS noise for a $1 \mathrm{~Hz}$ bandwidth. The signal for that value of optical loading is the mean value of the results. We then stepped the optical loading by an amount $\Delta P$ and repeated the process. The responsivity of the device is the derivative of the signal with respect to the optical power, and the NEP is simply $\Delta P$ divided by the signal-to-noise ratio. Figure 5(b) shows the data obtained in this manner.

Figure 6(a) shows a histogram of the measured NEPs; they cluster around $5 \times 10^{-20} \mathrm{~W} / \mathrm{Hz}^{1 / 2}$. Comparing the measured NEPs with the expected photon shot-noise NEP, we obtain the efficiency map shown in Fig. 6(b). Note that since not all resonances were found, the location of each

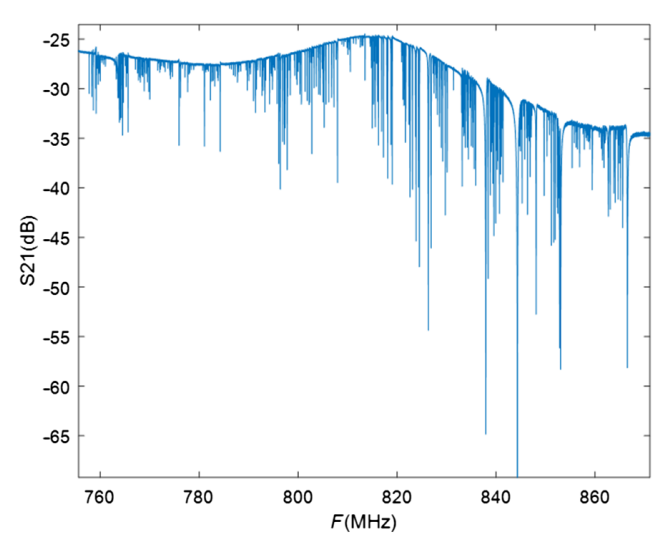

(a)

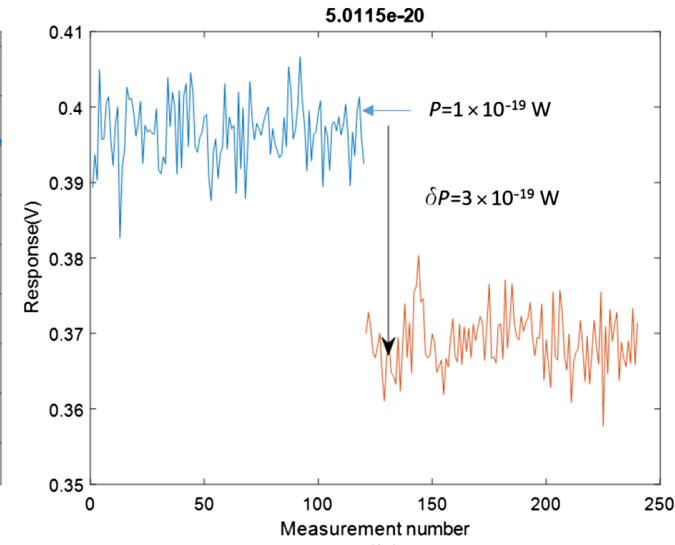

(b)

Fig. 5 (a) Transmission through QCD feedline. (b) Example of a NEP measurement. 


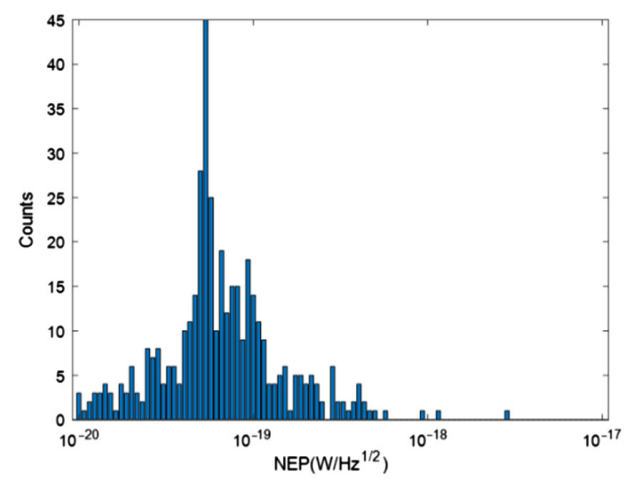

(a)

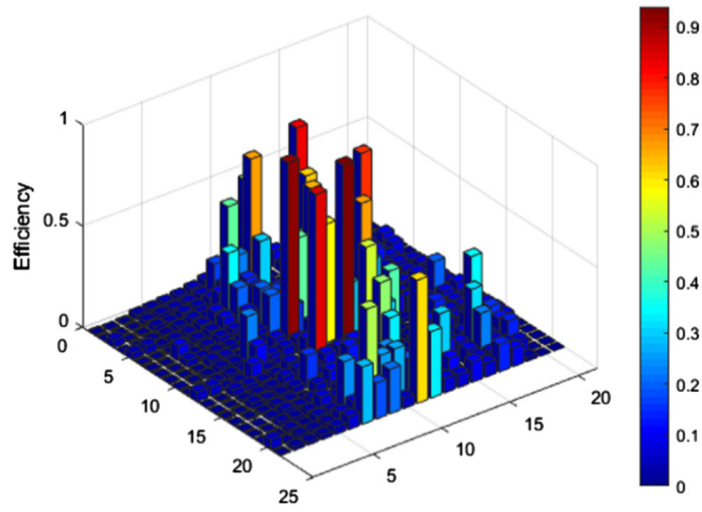

(b)

Fig. 6 (a) Histogram of measred NEPs. (b) Map of efficiencies calculated by comparing the measured NEPs with photon shot noise NEP.

resonance on the device may not be accurate. However, in general, we do expect the edges of the array to have poor efficiency simply due to the fact the edges are not illuminated because the diameter of the cylindrical entrance baffle is smaller than the array diameter. We will correct this in the near future by introducing larger diameter mesh filters.

\section{Path Forward}

\subsection{Uniformity}

The NEP measurements presented here demonstrate that the QCD has the potential to satisfy the OST requirements in a large format array. Even though the tests were performed at $200 \mu \mathrm{m}$ wavelength, simulations show the performance should satisfy the requirements of OST for wavelengths between 25 and $460 \mu \mathrm{m}$ as shown in Fig. 7. However, QCDs also display a large variability in performance that has to be addressed. We believe a source of this variability is the spread in tunnel junction resistances. We are addressing this issue by fabricating arrays of test tunnel junctions that can be probed at room temperature. We have initially seen a large variability in resistance measurements, but are converging on fabrication parameters which have already brought the yield of $100 \times 100 \mathrm{~nm}^{2}$ junctions to $70 \%$ and $100 \times 150 \mathrm{~nm}^{2}$ to $90 \%$. We are

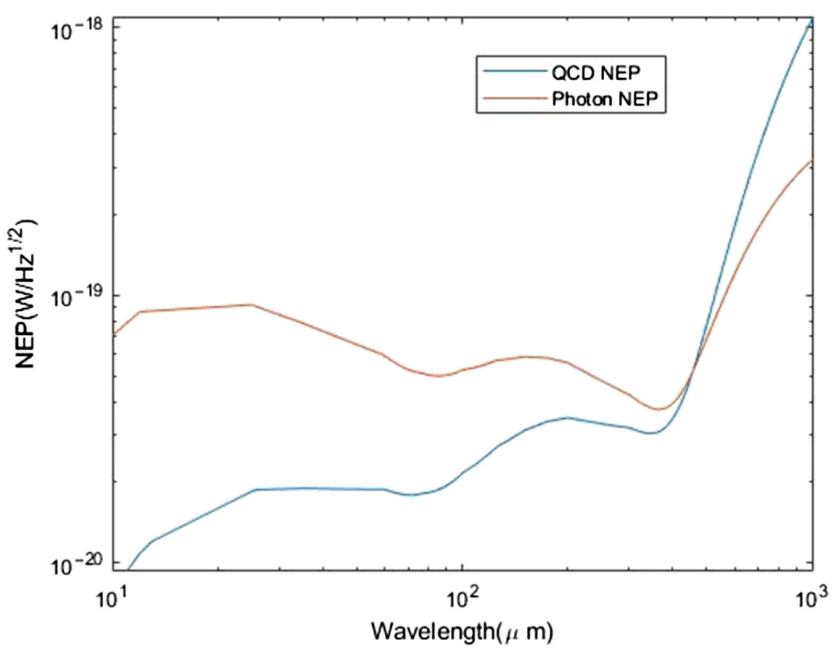

Fig. 7 Simulation of QCD NEP (blue) versus wavelength for the optical loading predicted for the OST compared with the required NEP (orange). 
working on improving the yield of these $100 \times 100 \mathrm{~nm}^{2}$ junctions further. We note that our simulations indicate that we could also use the higher-yield $100 \times 150 \mathrm{~nm}^{2}$ junctions to achieve shot-noise-limited performance, but this has to be confirmed experimentally.

Another issue that arose with the low-frequency design utilized for this latest 441-pixel prototype is a long-term fluctuation of the quantum capacitance signal. On the time scale of minutes, in some pixels, the quantum capacitance signal jumps between two values. This behavior was not present on the previous QCD arrays which operated with higher readout frequencies, so we believe the effect is due to the resonator design. To achieve the low readout frequencies (700 to $900 \mathrm{MHz}$ ), the resonator inductors (which are the frequency-determining elements of the pixels), must be large. They are composed of $1-\mu \mathrm{m}$-wide lines tightly meandered with only $1-\mu \mathrm{m}$ spacing. This is compared to the $4-\mu \mathrm{m}$ wide, $4-\mu \mathrm{m}$ spacing used for the higher frequency ( $\sim 4 \mathrm{GHz})$ designs. We believe this tight packing increases the likelihood of a charge fluctuator located between the lines to couple to the resonator and produce frequency noise. We have designed and fabricated $21 \times 21$ arrays with higher frequency readout with the wider inductor lines and spacing to test this hypothesis. These devices will be tested shortly.

\subsection{Dynamic Range}

An important consideration for Origins detectors is the dynamic range. The QCD loses sensitivity at high-incident power because the photon rates exceed the quasiparticle tunneling rates, increasing the device noise above the photon shot noise. Figure 8 illustrates this effect observed in devices described in Ref. 15. Figure 8(a) shows the response (amplitude of quantum capacitance) versus optical power for the mesh absorber device of Ref. 15 with island volume $0.022 \mu \mathrm{m}^{3}$ and absorber $1.5 \mu \mathrm{m}^{3}$. Figure 8(b) shows the NEP as a function of optical power for the same device. The region where shot noise dominates ranges at most from $10^{-20}$ to $10^{-18} \mathrm{~W}$. This is predicted by the simulations as shown in Fig. 8(c) (the blue circles). Simulations also predict that for a device with island volume $0.001 \mu \mathrm{m}^{3}$, absorber $0.044 \mu \mathrm{m}^{3}$ and with increased width of the junction from 0.1 to $0.15 \mu \mathrm{m}$ the shot noise limited region will extend from $10^{-22}$ to $2 \times 10^{-16} \mathrm{~W}$ (red dots). Even though there are many competing factors determining the NEP, the main reason for this is the fact that small island and reservoir volumes increase both tunneling in and tunneling out rates causing a decrease in the shot noise of electron tunneling, the main source of noise for the QCD. Furthermore, somewhat counter intuitively, increasing the width of the junction from 0.1 to $0.15 \mu \mathrm{m}$ also increases the tunneling rates due to a decreased tunneling resistance, whereas the reduced charging energy causes only a modest decrease in responsivity. Although we are at the lower limit for a mesh absorber volume, using an antenna coupled device allows for an absorber with this small volume and also a small volume island. We will explore devices with small island and absorber in the near future with the hope to increase the dynamic range.

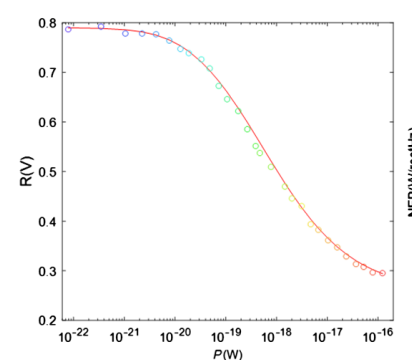

(a)

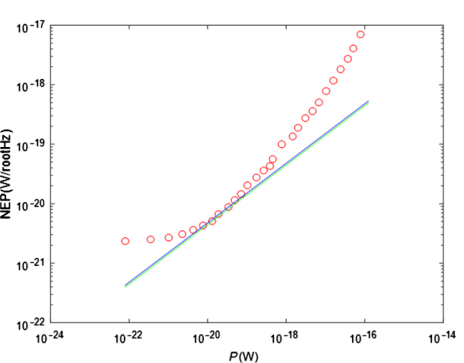

(b)

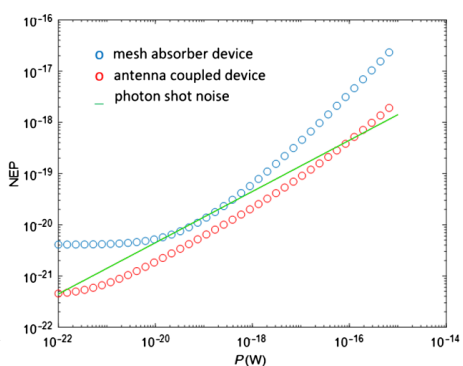

(c)

Fig. 8 (a) Response and (b) NEP versus optical power for the same device of Ref. 15. Note small region of shot noise limited performance; (c) simulations of NEP versus optical power for a mesh absorber with same parameters as the one from (a), and for an antenna coupled device with small island and absorber, and $0.15-\mu \mathrm{m}$ wide junction. 


\section{Acknowledgments}

We thank Richard E. Muller for performing electron beam lithography. This work was performed at the Jet Propulsion Laboratory, California Institute of Technology, under a contract with the National Aeronautics and Space Administration. Copyright 2020 California Institute of Technology. U.S. Government sponsorship acknowledged.

\section{References}

1. “Origins Space Telescope: Documents," https://asd.gsfc.nasa.gov/firs/docs/.

2. P. K. Day et al., "A broadband superconducting detector suitable for use in large arrays," Nature 425(6960), 817-821 (2003).

3. P. Khosropanah et al., "Low thermal conductance transition edge sensor (TES) for SPICA," AIP Conf. Proc. 1185, 42-47 (2009).

4. D. Morozov et al., "Ultrasensitive TES bolometers for space based FIR astronomy," AIP Conf. Proc. 1185, 48 (2009).

5. A. D. Beyer et al., "Characterizing SixNy absorbers and support beams for far-infrared/ submillimeter transition-edge sensors," Proc. SPIE 7741, 774121 (2010).

6. J. Wei et al., "Ultrasensitive hot-electron nanobolometers for terahertz astrophysics," Nat. Nanotechnol. 3(8), 496-500 (2008).

7. M. D. Shaw et al., "Quantum capacitance detector: a pair-breaking radiation detector based on the single Cooper-pair box," Phys. Rev. B 79(14), 144511 (2009).

8. J. Bueno et al., "Proof of concept of the quantum capacitance detector," Appl. Phys. Lett. 96(10), 103503 (2010).

9. J. Bueno et al., "Optical characterization of the quantum capacitance detector at $200 \mu \mathrm{m}$," Appl. Phys. Lett. 99(17), 173503 (2011).

10. K. J. Stone et al., "Real time quasiparticle tunneling measurements on an illuminated quantum capacitance detector," Appl. Phys. Lett. 100(26), 263509 (2012).

11. P. M. Echternach et al., "Photon shot noise limited detection of terahertz radiation using a quantum capacitance detector," Appl. Phys. Lett. 103(5), 053510 (2013).

12. B. S. Karasik and R. Cantor, "Demonstration of high optical sensitivity in far-infrared hot-electron bolometer," Appl. Phys. Lett. 98(19), 193503 (2011).

13. A. D. Beyer et al., "Effect of $\mathrm{Mo} / \mathrm{Cu}$ superconducting bilayer geometry on ultra-sensitive transition-edge sensor performance," IEEE Trans. Appl. Supercond. 23(3), 2100104 (2013).

14. S. J. C. Yates et al., "Photon noise limited radiation detection with lens-antenna coupled microwave kinetic inductance detectors," Appl. Phys. Lett. 99(7), 073505 (2011).

15. P. M. Echternach et al., "Single photon detection of $1.5 \mathrm{THz}$ radiation with the quantum capacitance detector," Nat. Astron. 2(1), 90-97, (2018).

16. T. Duty et al., "Observation of quantum capacitance in the Cooper-Pair transistor," Phys. Rev. Lett. 95(20), 206807 (2005).

17. G. Clarke et al., "Superconductive bolometers for submillimeter wavelengths," J. Appl. Phys. 48, 4865-4879 (1977).

18. M. J. Persky, "Review of black surfaces for space-borne infrared systems," Rev. Sci. Instrum. 70, 2193-2217 (1999).

Biographies of the authors are not available. 